\title{
Gilteritinib versus chemotherapy in Japanese patients with FLT3-mutated relapsed/refractory acute myeloid leukemia
}

\author{
Naoko Hosono ${ }^{1}$ (1) $\cdot$ Hisayuki Yokoyama ${ }^{2,24} \cdot$ Nobuyuki Aotsuka $^{3} \cdot$ Kiyoshi Ando $^{4} \cdot$ Hiroatsu lida $^{5} \cdot$ Takayuki Ishikawa $^{6}$. \\ Kensuke Usuki $^{7} \cdot$ Masahiro Onozawa $^{8} \cdot$ Masahiro Kizaki $^{9} \cdot$ Kohmei Kubo $^{10}$. Junya Kuroda ${ }^{11} \cdot$ Yukio Kobayashi $^{12}$. \\ Takayuki Shimizu $^{13} \cdot$ Shigeru Chiba $^{14} \cdot$ Miho Nara $^{15} \cdot$ Tomoko Hata $^{16} \cdot$ Michihiro Hidaka $^{17}$. Shin-Ichiro Fujiwara ${ }^{18}$. \\ Yoshinobu Maeda ${ }^{19}$. Yasuyoshi Morita ${ }^{20}$. Mikiko Kusano ${ }^{21} \cdot$ Qiaoyang Lu $^{22}$. Shuichi Miyawaki ${ }^{23} \cdot$ Erhan Berrak $^{22}$. \\ Nahla Hasabou ${ }^{22} \cdot$ Tomoki Naoe $^{5}$
}

Received: 30 April 2021 / Accepted: 26 July 2021 / Published online: 7 August 2021

(c) The Author(s) 2021

\begin{abstract}
Background Until recently, no effective targeted therapies for FLT3-mutated (FLT3 ${ }^{\text {mut+)}}$ relapsed/refractory (R/R) acute myeloid leukemia (AML) were available in Japan. The FLT3 inhibitor, gilteritinib, was approved in Japan for patients with FLT3 ${ }^{\text {mut }}$ R/R AML based on the phase 3 ADMIRAL trial, which demonstrated the superiority of gilteritinib over salvage chemotherapy (SC) with respect to overall survival (OS; median OS, 9.3 vs 5.6 months, respectively; hazard ratio, 0.64 [95\% confidence interval 0.49, 0.83]; $P<0.001$ ).

Methods We evaluated the Japanese subgroup $(n=48)$ of the ADMIRAL trial, which included 33 patients randomized to $120-\mathrm{mg} /$ day gilteritinib and 15 randomized to SC.

Results Median OS was 14.3 months in the gilteritinib arm and 9.6 months in the SC arm. The complete remission/complete remission with partial hematologic recovery rate was higher in the gilteritinib arm (48.5\%) than in the SC arm (13.3\%). After adjustment for drug exposure, fewer adverse events (AEs) occurred in the gilteritinib arm than in the SC arm. Common grade $\geq 3$ AEs related to gilteritinib were febrile neutropenia (36\%), decreased platelet count (27\%), and anemia (24\%). Conclusion Findings in Japanese patients are consistent with those of the overall ADMIRAL study population.
\end{abstract}

Keywords Acute myeloid leukemia · FLT3 inhibitor · FLT3 mutations

Naoko Hosono

hosono@u-fukui.ac.jp

1 Department of Hematology and Oncology, Faculty of Medical Sciences, University of Fukui, Fukui 910-1193, Japan

2 Sendai Medical Center, Sendai, Japan

3 Japanese Red Cross Narita Hospital, Narita, Japan

4 Tokai University School of Medicine, Isehara, Japan

5 NHO Nagoya Medical Center, Nagoya, Japan

6 Kobe City Medical Center General Hospital, Hyogo, Japan

7 NTT Medical Center Tokyo, Tokyo, Japan

8 Hokkaido University, Sapporo, Japan

9 Saitama Medical Center, Saitama Medical University, Kawagoe, Japan

10 Aomori Prefectural Central Hospital, Aomori, Japan

11 Kyoto Prefectural University of Medicine, Kyoto, Japan
12 International University of Health and Welfare (IUHW), Mita Hospital, Tokyo, Japan

13 Keio University School of Medicine, Tokyo, Japan

14 University of Tsukuba, Tsukuba, Japan

15 Akita University, Akita, Japan

16 Nagasaki University, Nagasaki, Japan

17 Kumamoto Medical Center, Kumamoto, Japan

18 Jichi Medical University, Shimotsuke, Japan

19 Okayama University Hospital, Okayama, Japan

20 Kindai University, Osaka, Japan

21 Astellas Pharma, Inc., Tokyo, Japan

22 Astellas Pharma US, Inc., Northbrook, IL, USA

23 Tokyo Metropolitan Ohtsuka Hospital, Tokyo, Japan

24 Present Address: Tohoku University, Sendai, Japan 


\section{Introduction}

Acute myeloid leukemia (AML) is the most common leukemia among Japanese adults, and accounts for approximately $70 \%$ of all myeloid leukemias [1,2]. Patients with relapsed AML have a poor prognosis, with a 5-year survival rate of $10-11 \%[3,4]$. Activating mutations in fmslike tyrosine kinase 3 (FLT3) occur in approximately $30 \%$ of patients with AML $[5,6]$, primarily as internal tandem duplication (FLT3-ITD) mutations in the juxtamembrane region $(\sim 20-25 \%)$ and missense point mutations in the tyrosine kinase domain (FLT3-TKD; 5-7\%) [5, 7-11]. In AML, FLT3-ITD mutations confer a strong negative impact on survival[12-14]; FLT3-TKD point mutations have been implicated in resistance to FLT3 inhibitors [15, 16].

Several FLT3 tyrosine kinase inhibitors (TKIs) are under development or have been approved for the treatment of FLT3-mutated $\left(F L T 3^{\mathrm{mut}+}\right)$ AML. First-generation multitargeted FLT3 TKIs (sunitinib, sorafenib, midostaurin, lestaurtinib, ponatinib) [17] lacked specificity for FLT3-ITD, resulting in short-lived antileukemic activity, especially as single agents [18-21]. Second-generation FLT3 TKIs (gilteritinib and quizartinib) boasting higher potency and greater specificity for FLT3 were subsequently developed [17]. While quizartinib demonstrated single-agent activity in relapsed/refractory (R/R) FLT3-ITD-positive (FLT3-ITD+) AML patients [22], treatment-emergent FLT3-TKD mutations leading to drug resistance [15] and myelosuppression due to quizartinib's effects on c-kit [23] are of concern.

Gilteritinib is a highly selective, oral FLT3 inhibitor with activity against both FLT3-ITD and -TKD mutations with only weak activity against c-Kit $[24,25]$. The phase $1 / 2$ CHRYSALIS study demonstrated that single-agent gilteritinib had a favorable safety profile and, at $\geq 80-\mathrm{mg} /$ day doses, induced $\geq 90 \%$ inhibition of FLT3 autophosphorylation with a composite complete remission (CRc) rate of $41 \%$ in patients with R/R FLT3 ${ }^{\mathrm{mut}+}$ AML [26]. A starting dose of $120 \mathrm{mg} /$ day was recommended for further study [26].

Gilteritinib was the first FLT3 TKI approved by the Ministry of Health Labor and Wealth (MHLW) in Japan for patients with R/R FLT3 ${ }^{\text {mut+ }}$ AML [27]. Approval was based on interim results from the phase 3 ADMIRAL study (NCT02421939) of gilteritinib versus salvage chemotherapy (SC) in patients with R/R FLT3 ${ }^{\text {mut+ }}$ AML, where 40 of $142(28 \%)$ patients in the gilteritinib arm had achieved complete remission (CR) or CR with partial hematologic recovery (CRh) [27]. Final results from ADMIRAL demonstrated significantly longer overall survival (OS) with gilteritinib than with SC (9.3 vs 5.6 months, respectively; hazard ratio [HR] for death, $0.64 ; 95 \%$ confidence interval [CI] 0.49, 0.83; $P<0.001)$ [28].
As treatment efficacy and safety may vary by ethnicity, evaluation of gilteritinib in Japanese patients with R/R FLT$3^{\text {mut+ }}$ AML is warranted. The safety and antitumor effects of gilteritinib were reported in a phase 1 study of 24 Japanese patients with R/R AML; however, only 3 FLT3 ${ }^{\text {mut+ }}$ Japanese patients had received $\geq 80$-mg gilteritinib [29]. The ADMIRAL trial included $54 \mathrm{R} / \mathrm{R} F L T 3^{\text {mut+ }}$ AML patients from Asian countries, with most (89\%; 48 of 54) being Japanese. A subgroup analysis was conducted to confirm the clinical risk-benefit profile of gilteritinib in Japanese patients enrolled in the ADMIRAL trial.

\section{Materials and methods}

\section{Trial design and oversight}

This randomized phase 3, open-label study (NCT02421939; ADMIRAL) was conducted at 107 centers in 14 countries [28]. Japanese patients were enrolled at 20 sites across Japan. The trial was reviewed and approved by site-specific institutional review boards or ethics committees and conducted according to the principles of the Declaration of Helsinki. All patients provided written informed consent at enrollment. All authors had access to study data and have ascertained its accuracy and adherence to the study protocol.

\section{Patients}

Complete details of the ADMIRAL population are provided in the primary publication [28]. Adults with AML were eligible if they were refractory to one or two cycles of conventional anthracycline-containing induction therapy or were experiencing hematologic relapse after first CR. Patients ineligible for anthracycline-containing induction regimens could participate if they had completed $\geq 1$ cycle of alternative standard therapy judged as the optimum choice to induce remission. At enrollment, patients' marrow and blood were screened for FLT3 mutations by a central laboratory. Enrollment based on local FLT3 mutation testing was permitted in patients with rapidly proliferative disease. Patients were required to have either FLT3-ITD or FLT3-TKD D835 or I836 mutations (Invivoscribe; San Diego, CA) using a polymerase chain reaction-based assay modeled on published methods (LeukoStrat ${ }^{\circledR} \mathrm{CDx}$ ) [30].

\section{Randomization and treatments}

A brief description of the ADMIRAL study design, randomization, and treatment are described (Supplement, Figure S1), with further details in the primary publication [28]. Briefly, enrolled patients were randomized 2:1 to receive 28 -day cycles of once-daily gilteritinib $(120 \mathrm{mg})$ or 
preselected high- or low-intensity SC. High- or low-intensity SC regimens were selected by study investigators before randomization from the following options: high-intensity SC: mitoxantrone, etoposide, and cytarabine (MEC)[31], or fludarabine, cytarabine, granulocyte-colony stimulating factor, and idarubicin (FLAG-IDA)[32]; or low-intensity SC: low-dose cytarabine (LoDAC) or azacitidine (AZA). High-intensity chemotherapy was administered for one to two cycles; gilteritinib or low-intensity SC were administered until documented lack of clinical benefit, intolerance, or other protocol-defined discontinuation criterion [28].

\section{Endpoints and assessments}

The co-primary endpoints were OS and the rates of CR/CRh, where $\mathrm{CRh}$ was defined as $\mathrm{CR}$ with a platelet count of $>0.5$ $\mathrm{Gi} / \mathrm{L}$ and an absolute neutrophil count of $>0.5 \mathrm{Gi} / \mathrm{L}$. Key secondary endpoints were event-free survival (EFS) and CR rate, where EFS was defined as the time from the date of randomization until the date of documented relapse (excludes relapse after partial remission), treatment failure, or death. The CR/CRh rate was evaluated in the first interim analysis in the gilteritinib arm only and was also summarized in the final analysis for both treatment arms. Overall survival, EFS, CR rate, and other response outcomes were evaluated in the final analysis. Treatment response was assessed using the modified International Working Group criteria [33]; best response was captured at any postbaseline visit. Treatment-emergent adverse events (TEAEs) were graded according to the Common Terminology Criteria for Adverse Events (v4.03). The exposure-adjusted incidence of TEAEs per patient-year (PY) was calculated by dividing exposure duration in years by the number of patients in each treatment arm. Postbaseline (i.e., 29 days after first dose until the last dose) transfusion status was evaluated in patients on gilteritinib therapy for $\geq 84$ days; transfusion independence was achieved if no red blood cell/platelet transfusions were administered for 56 consecutive days during the postbaseline period. The incidence and duration of hospitalization were also evaluated.

\section{Statistical analysis}

Complete details on statistical analyses in the ADMIRAL trial have been previously published [28]. The CR/CRh rate in the gilteritinib arm was assessed at the first planned interim analysis when approximately 141 patients in the intention-to-treat (ITT) population had reached 112 days (four treatment cycles) after randomization or the first dose; interim evaluation of $\mathrm{CR} / \mathrm{CRh}$ rate had no impact on trial conduct [28].
The Japanese subgroup was analyzed similarly to the overall ITT population. The Kaplan-Meier method combined with the Greenwood formula was used to determine OS and EFS and corresponding 95\% CIs. As the trial was designed to test hypotheses in the overall ITT population, it was not powered to detect cross-arm differences in this subgroup analysis.

\section{Results}

\section{Patient disposition}

Of the 625 patients screened from October 2015 to February 2018, 371 (ITT population) were randomized to gilteritinib $(n=247)$ or SC $(n=124)$, which included 48 Japanese patients (gilteritinib, $n=33$; SC, $n=15$ ) (Fig. 1). At the time of primary analysis (September 17, 2018), 24 of 48 (50\%) Japanese patients remained alive $(n=15 / 33$, gilteritinib; $n=9 / 15$, SC) and 7 remained on gilteritinib therapy. In the Japanese subgroup, $79 \%(n=11 / 14)$ of patients in the SC arm received low-intensity chemotherapy compared with $38 \%(n=41 / 109)$ of SC-treated patients in the overall safety analysis set (SAF) population.

\section{Demographic and baseline characteristics}

As shown in Table 1, 46\% $(n=22 / 48)$ of patients had relapsed AML (gilteritinib, 52\% [ $n=17 / 33]$; SC, 33\% $[n=5 / 15])$ and $54 \%(n=26 / 48)$ had primary refractory disease (gilteritinib, $48 \%$ [ $n=16 / 33]$; SC, $67 \%$ [ $n=10 / 15]$ ). The proportion of Japanese patients aged $\geq 65$ years was higher in the SC arm $(n=13 / 15 ; 87 \%)$ than in the gilteritinib arm $(n=15 / 33 ; 45 \%)$. Japanese patients had lower body weight (mean \pm standard deviation [SD]: $52.91 \pm 11.10 \mathrm{~kg}$ ) compared with the overall ITT population (mean \pm SD: $71.82 \pm 20.25 \mathrm{~kg}$ ).

\section{Study drug exposure and dose modifications}

The median duration of exposure to gilteritinib in the Japanese subgroup was 5.1 months

(154 days) (Table 2), which was longer than that reported for the overall SAF population (4.1 months); the median duration of exposure to $\mathrm{SC}$ was the same in both populations (0.9 months). Overall, 33\% of gilteritinib-treated patients $(n=11 / 33)$ in the Japanese subgroup had dose increases to $200 \mathrm{mg} /$ day (Table 2), which was similar to the overall SAF population (32\%). Gilteritinib dose reductions were slightly higher in the Japanese subgroup (39\%; $n=13 / 33)$ than in the overall SAF population (31\%); dose interruptions were also higher in the Japanese subgroup (67\% vs 50\%, respectively). 


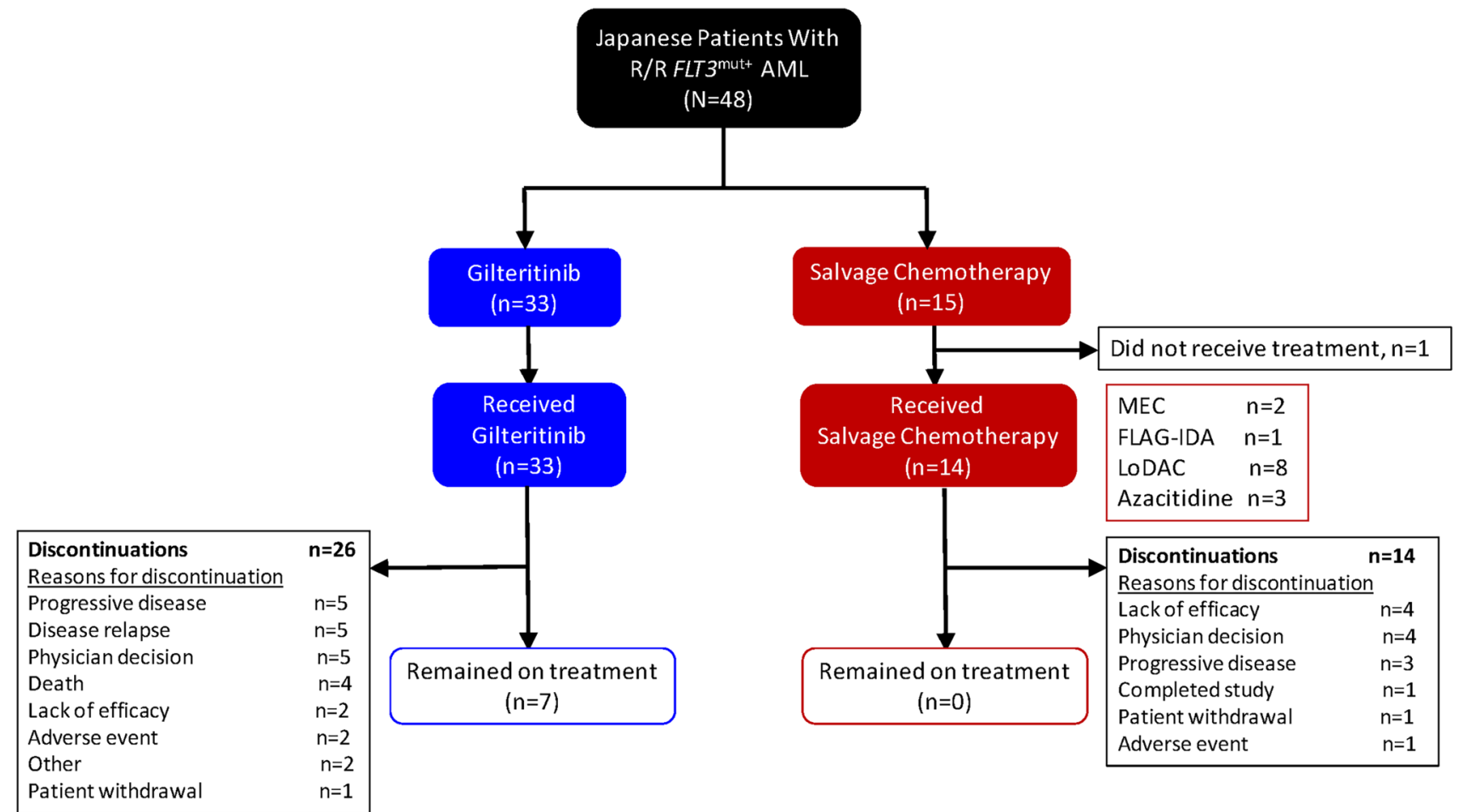

Fig. 1 Patient disposition: Japanese ITT population. $A M L$ acute myeloid leukemia, FLAG-IDA fludarabine, cytarabine, and idarubicin plus granulocyte colony-stimulating factor, ITT intention-to-treat,

\section{Survival outcomes}

In the overall ITT population, median OS was 9.3 months in the gilteritinib arm and 5.6 months in the SC arm; 1-year survival rates were $37 \%$ for patients who received gilteritinib compared with $17 \%$ for patients who received SC. In the Japanese subgroup, the median OS was 14.3 months in gilteritinib-treated patients and 9.6 months in patients treated with SC (Fig. 2); 1-year survival rates were 54.3\% in the gilteritinib arm versus $26.3 \%$ in the SC arm.

\section{Response outcomes}

In the overall ITT population, the rate of CR/CRh was higher in the gilteritinib arm than in the chemotherapy arm (34.0\% vs $15.3 \%$, respectively), as was the CR rate $(21.1 \%$ vs $10.5 \%$, respectively). Similarly, CR/CRh rates in the Japanese subgroup were also higher in the gilteritinib arm (48.5\%) than in the SC arm (13.3\%) (Table 3). Rates of CR/CRh before HSCT in the Japanese subgroup gilteritinib and SC arms were $30.3 \%$ and $13.3 \%$, respectively, and were comparable to the overall ITT population (gilteritinib, 26.3\%; SC, 15.3\%); CR rates (gilteritinib, 24.2\%; SC, 6.7\%) in the Japanese subgroup were also similar to the overall ITT population (gilteritinib, $21.1 \%$; SC, $10.5 \%$ ). Median durations of CR/CRh or
LoDAC low-dose cytarabine, $M E C$ mitoxantrone, etoposide, and cytarabine, $m u t+$ mutated, $R / R$ relapsed or refractory

$\mathrm{CR}$ in the gilteritinib arm were not reached; the median duration of CRc in the gilteritinib arm was 9.2 months. Median durations of $\mathrm{CR} / \mathrm{CRh}, \mathrm{CR}$, and $\mathrm{CRc}$ were not evaluable in the SC arm. In the overall ITT population, median durations of $\mathrm{CR}, \mathrm{CR} / \mathrm{CRh}$, and $\mathrm{CRc}$ in the gilteritinib arm were $14.8,11.0$, and 4.6 months, respectively; median durations of $\mathrm{CR}$ and $\mathrm{CR} / \mathrm{CRh}$ in the $\mathrm{SC}$ arm were both 1.8 months; the median duration of $\mathrm{CRc}$ was not evaluable.

\section{Transplantation rates}

In the overall ITT population, more patients assigned to gilteritinib $(n=63 / 247 ; 25.5 \%)$ than those assigned to SC ( $n=19 / 124 ; 15.3 \%)$ underwent HSCT during the study. In the Japanese subgroup, 12 patients $(36.4 \%)$ assigned to gilteritinib and $2(13.3 \%)$ assigned to SC underwent HSCT. Nine Japanese patients who underwent HSCT after initial gilteritinib treatment resumed gilteritinib after transplantation.

\section{Safety and tolerability}

Most patients in the Japanese SAF $(N=47)$ experienced TEAEs (Table 4; gilteritinib, $100 \%$ [ $n=33 / 33]$; SC, $93 \%$ $[n=13 / 14]$ ). Drug-related TEAEs occurred in $88 \%$ of 
Table 1 Patient demographics and disposition (Japan ITT population; $N=48$ )

\begin{tabular}{|c|c|c|c|}
\hline Characteristic & Gilteritinib $(n=33)$ & $\begin{array}{l}\text { Salvage chemo- } \\
\text { therapy }(n=15)\end{array}$ & $\begin{array}{l}\text { Total } \\
(N=48)\end{array}$ \\
\hline Median age, years (range) & $60.0(22-84)$ & $69.0(28-79)$ & $67.5(22-84)$ \\
\hline \multicolumn{4}{|l|}{ Sex, $n(\%)$} \\
\hline Male & $14(42)$ & $6(40)$ & $20(42)$ \\
\hline Female & $19(58)$ & $9(60)$ & $28(58)$ \\
\hline \multicolumn{4}{|l|}{ Age group ${ }^{\mathrm{a}}, n(\%)$} \\
\hline$<65$ years & $18(55)$ & $2(13)$ & $20(42)$ \\
\hline$\geq 65$ years & $15(45)$ & $13(87)$ & $28(58)$ \\
\hline \multicolumn{4}{|l|}{ ECOG performance status, $n(\%)$} \\
\hline $0-1$ & $30(91)$ & $14(93)$ & $44(92)$ \\
\hline$\geq 2$ & $3(9)$ & $1(7)$ & $4(8)$ \\
\hline \multicolumn{4}{|l|}{ Central FLT3 mutation status ${ }^{\mathrm{a}}, n(\%)$} \\
\hline FLT3-ITD only & $29(88)$ & $15(100)$ & $44(92)$ \\
\hline FLT3-TKD only & $3(9)$ & 0 & $3(6)$ \\
\hline FLT3-ITD and FLT3-TKD & $1(3)$ & 0 & $1(2)$ \\
\hline \multicolumn{4}{|l|}{ AML type, $n(\%)$} \\
\hline De novo & $31(94)$ & $15(100)$ & $46(96)$ \\
\hline Secondary & $2(6)$ & 0 & $2(4)$ \\
\hline \multicolumn{4}{|l|}{ Cytogenetic risk status $^{\mathrm{a}}, n(\%)$} \\
\hline Favorable & $2(6)$ & 0 & $2(4)$ \\
\hline Intermediate & $20(61)$ & $13(87)$ & $33(69)$ \\
\hline Unfavorable & $2(6)$ & $1(7)$ & $3(6)$ \\
\hline Other/unknown & $9(27)$ & $1(7)$ & $10(21)$ \\
\hline \multicolumn{4}{|l|}{ Response to first-line therapy, $n(\%)$} \\
\hline Primary refractory AML without HSCT & $16(48)$ & $10(67)$ & $26(54)$ \\
\hline Relapse $\leq 6$ months after allogeneic HSCT & $2(6)$ & 0 & $2(4)$ \\
\hline Relapse $>6$ months after allogeneic HSCT & $2(6)$ & 0 & $2(4)$ \\
\hline Relapse $\leq 6$ months after CRc and no HSCT & $7(21)$ & $3(20)$ & $10(21)$ \\
\hline Relapse $>6$ months after CRc and no HSCT & $6(18)$ & $2(13)$ & $8(17)$ \\
\hline \multicolumn{4}{|l|}{ Prior use of a FLT3 inhibitor, $n(\%)$} \\
\hline Yes & 0 & 0 & 0 \\
\hline No & $33(100)$ & $15(100)$ & $48(100)$ \\
\hline \multicolumn{4}{|l|}{ Previous HSCT, $n(\%)$} \\
\hline Yes & $4(12)$ & 0 & $4(8)$ \\
\hline No & $29(88)$ & $15(100)$ & $44(92)$ \\
\hline \multicolumn{4}{|l|}{ Preselected chemotherapy ${ }^{\mathrm{a}}, n(\%)$} \\
\hline High-intensity & $16(48)$ & $3(20)$ & $19(40)$ \\
\hline Low-intensity & $17(52)$ & $12(80)$ & $29(60)$ \\
\hline
\end{tabular}

Percentages were rounded to the nearest whole number

$A M L$ acute myeloid leukemia, $C R c$ complete composite remission, ECOG Eastern Cooperative Oncology Group, FLT3 fms-like tyrosine kinase 3, HSCT hematopoietic stem cell transplantation, ITD internal tandem duplication, TKD tyrosine kinase domain

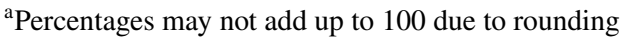

patients treated with gilteritinib and in $71 \%$ of patients treated with SC. Drug-related TEAEs leading to gilteritinib discontinuation occurred in three patients (hyperglycemia, intestinal ischemia, sepsis, back pain, depressed consciousness $[n=1]$; cholecystitis $[n=1]$; pneumonia $[n=1])$. Drugrelated TEAEs leading to discontinuation of SC occurred in one patient (febrile neutropenia, diabetes mellitus, and delirium). Serious drug-related TEAEs occurring in $\geq 2$ gilteritinib-treated patients were febrile neutropenia $(n=3)$, increased alanine aminotransferase (ALT; $n=3$ ), increased aspartate aminotransferase (AST; $n=3$ ), and pneumonia $(n=2)$. Five gilteritinib-treated patients died from TEAEs: AML progression $(n=2)$; intestinal ischemia, sepsis, depressed consciousness $(n=1)$; pancreatitis $(n=1)$; and 
Table 2 Study drug exposure and dose modifications (Japan SAF; $N=47$ )

\begin{tabular}{|c|c|c|c|c|c|}
\hline \multirow[t]{2}{*}{ Parameter } & \multirow{2}{*}{$\begin{array}{l}\text { Gilteritinib } \\
(n=33)\end{array}$} & \multicolumn{4}{|c|}{ Salvage chemotherapy $(n=14)$} \\
\hline & & $\operatorname{MEC}(n=2)$ & $\begin{array}{l}\text { FLAG-IDA } \\
(n=1)\end{array}$ & $\begin{array}{l}\text { Azacitidine } \\
(n=3)\end{array}$ & $\begin{array}{l}\text { LoDAC } \\
(n=8)\end{array}$ \\
\hline Median duration of exposure, days (range) & $154(37-595)$ & $28(28-28)$ & $28(28-28)$ & $28(28-111)$ & $28(28-28)$ \\
\hline \multicolumn{6}{|l|}{ Duration of exposure, $n(\%)$} \\
\hline$\geq 6$ to $<28$ days & 0 & 0 & 0 & 0 & 0 \\
\hline$\geq 28$ to $<84$ days & $9(27)$ & $2(100)$ & $1(100)$ & $2(67)$ & $8(100)$ \\
\hline$\geq 84$ to $<168$ days & $8(24)$ & 0 & 0 & $1(33)$ & 0 \\
\hline$\geq 168$ days & $16(48)$ & 0 & 0 & 0 & 0 \\
\hline Median number of treatment cycles (range) & $6(2-22)$ & $1(1-1)$ & $1(1-1)$ & $1(1-4)$ & $1(1-1)$ \\
\hline \multicolumn{6}{|l|}{ Dose modifications, $n(\%)$} \\
\hline Increases* & $11(33)$ & 0 & 0 & 0 & 0 \\
\hline Decreases* & $13(39)$ & 0 & 0 & 0 & 0 \\
\hline Interruptions & $22(67)$ & 0 & 0 & $1(33)$ & 0 \\
\hline
\end{tabular}

Percentages were rounded to the nearest whole number

FLAG-IDA fludarabine, cytarabine, idarubicin, and granulocyte colony-stimulating factor, LoDAC low-dose cytarabine, MEC mitoxantrone, etoposide, and cytarabine, $S A F$ safety analysis set

*One patient who had a dose increase from 120 to $200 \mathrm{mg}$ followed by a dose decrease to $120 \mathrm{mg}$ and a subsequent dose decrease from 120 to $80 \mathrm{mg}$

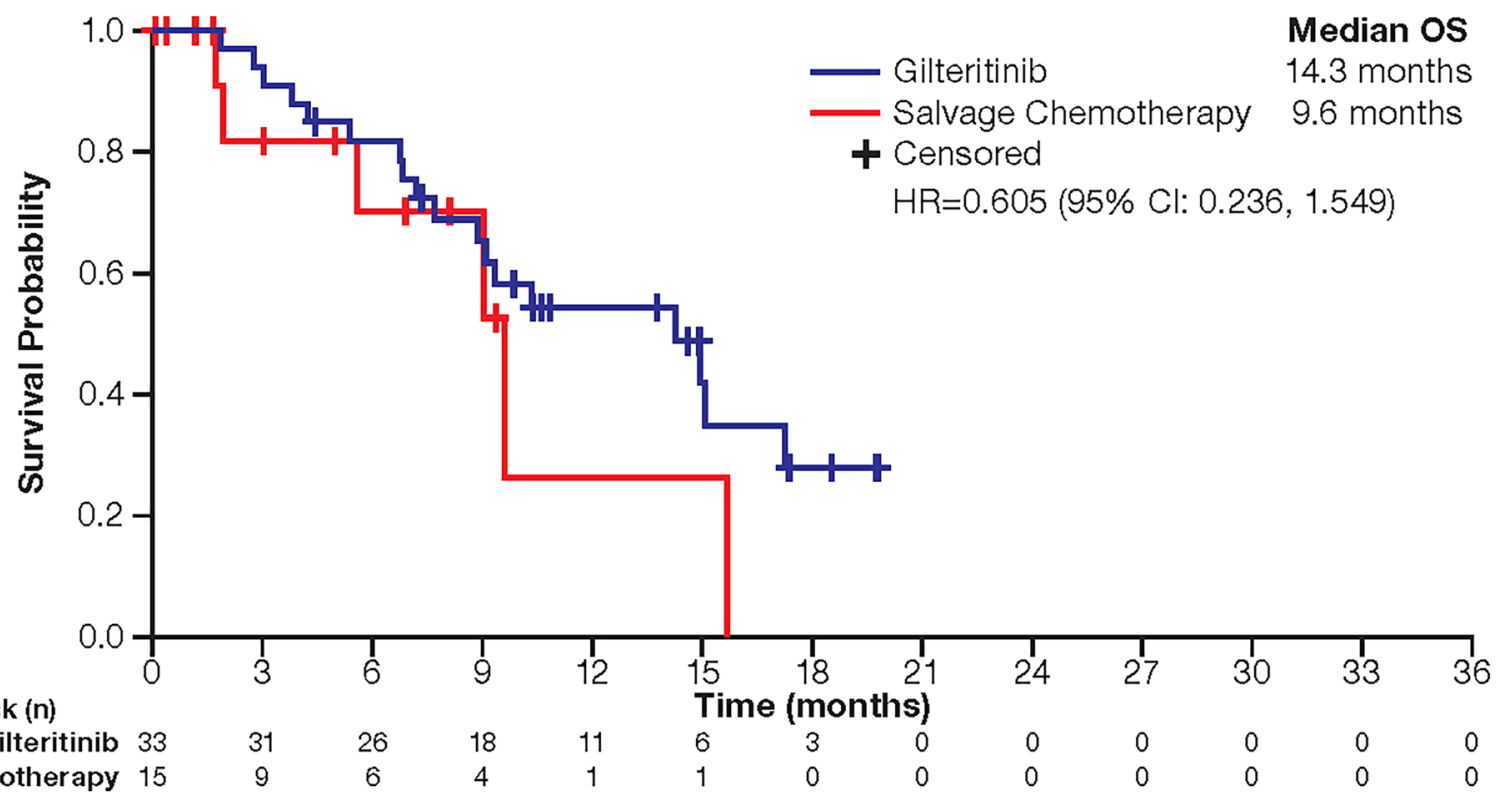

Fig. 2 Overall survival (Japan ITT population; $N=48$ ). $H R$ hazard ratio, ITT intention-to-treat, $O S$ overall survival

pneumonia $(n=1)$. Two deaths stemmed from drug-related TEAEs (intestinal ischemia, sepsis, depressed consciousness $[n=1]$; pneumonia $[n=1])$.

Gilteritinib was generally well tolerated in this Japanese subgroup. Although gilteritinib exposure was approximately five times longer than SC exposure, the exposure-adjusted incidence of TEAEs was lower with gilteritinib (53.84/PY) than with SC (93.08/PY; Table 4), including all drug-related
TEAEs leading to discontinuation (gilteritinib, 0.38/PY; SC, 2.31/PY) and grade $\geq 3$ drug-related TEAEs (gilteritinib, 6.27/PY; SC, 26.92/PY). In the Japanese subgroup, incidences of TEAEs (100\%) and deaths (55\%) in gilteritinibtreated patients were similar to those observed in the overall SAF population (100\% and $69 \%$, respectively).

The most common TEAEs in gilteritinib-treated patients were febrile neutropenia (55\%), increased AST (52\%), 
Table 3 Response outcomes (Japan ITT Population; $N=48$ )

\begin{tabular}{|c|c|c|}
\hline Response parameter & Gilteritinib $(n=33)$ & $\begin{array}{l}\text { Salvage } \\
\text { chemotherapy } \\
(n=15)\end{array}$ \\
\hline $\mathrm{CR}, n(\%)$ & $8(24.2)$ & $1(6.7)$ \\
\hline $\mathrm{CRp}, n(\%)$ & $3(9.1)$ & 0 \\
\hline $\mathrm{CRi}, n(\%)$ & $8(24.2)$ & $2(13.3)$ \\
\hline $\mathrm{CRh}, n(\%)$ & $8(24.2)$ & $1(6.7)$ \\
\hline $\mathrm{CR} / \mathrm{CRh}, n(\%)$ & $16(48.5)$ & $2(13.3)$ \\
\hline $\mathrm{CRc}^{\mathrm{a}}, n(\%)$ & $19(57.6)$ & $3(20.0)$ \\
\hline $\mathrm{PR}, n(\%)$ & $5(15.2)$ & $1(6.7)$ \\
\hline $\mathrm{NR}, n(\%)$ & $9(27.3)$ & $5(33.3)$ \\
\hline $\mathrm{ORR}^{\mathrm{b}}, n(\%)$ & $24(72.7)$ & $4(26.7)$ \\
\hline NE, $n(\%)$ & 0 & $6(40.0)$ \\
\hline
\end{tabular}

$C R$ complete remission, $C R c$ composite complete remission, $C R h$ complete remission with partial hematologic recovery, $C R i$ complete remission with incomplete hematologic recovery, $C R p$ complete remission with incomplete platelet recovery, ITT intention-to-treat, $N E$ not evaluable, $N R$ no response, $O R R$ overall response rate, $P R$ partial remission

${ }^{a}$ Defined as CR plus CRi plus CRp

${ }^{\mathrm{b}}$ Defined as CRc plus PR

increased ALT (46\%), anemia (39\%), constipation (39\%), increased blood creatinine phosphokinase (39\%), decreased platelet count (39\%), and nausea (36\%) (Table 5). Most common TEAEs in patients treated with SC were anemia (57\%), decreased platelet count (50\%), and decreased white blood cell count (36\%). Higher incidences of grade $\geq 3$ febrile neutropenia $(55 \%)$ and neutropenia $(12 \%)$ were seen with gilteritinib versus SC (29\% and 7\%, respectively). Lower incidences of grade $\geq 3$ anemia (33\%) and thrombocytopenia
(9\%) were observed with gilteritinib versus SC (57\% and $21 \%$, respectively).

Most common grade $\geq 3$ drug-related TEAEs observed with gilteritinib treatment in the Japanese subgroup were febrile neutropenia (36\%), decreased platelet count (27\%), and anemia (24\%) (Table 6). When comparing the Japanese subgroup to all other ADMIRAL patients, no clinically significant TEAEs were unique to Japanese patients.

Overall, seven gilteritinib-treated patients and two SCtreated patients in the Japanese subgroup had a maximum postbaseline mean Fridericia-corrected QT interval (QTcF) increase of $>30 \mathrm{~ms}$; two patients treated with gilteritinib had a maximum postbaseline mean QTcF increase of $>60 \mathrm{~ms}$. One case of prolonged QTcF occurred with gilteritinib. As no maximum postbaseline QTcF increases of $>500 \mathrm{~ms}$ occurred, no gilteritinib dose reductions or interruptions were required. No cases of Torsade de Pointes were observed in the entire SAF population.

\section{Transfusion status and hospitalization in patients treated with gilteritinib}

Among gilteritinib-treated Japanese patients, 22 were transfusion-dependent (TD) and 11 were transfusion-independent (TI) at baseline. Six of 22 TD patients (27\%) at baseline became TI during the postbaseline period. Eight of 11 (73\%) TI patients at baseline remained TI postbaseline.

Hospitalization rates during gilteritinib therapy were $94 \%$ in the Japanese subgroup and $85 \%$ in the overall ITT population; most hospitalizations ( 96\%) in both populations were outside the intensive care unit. While Japanese patients had longer hospitalization duration (median, 133 days; range 10-497 days) than the overall ITT population (median,
Table 4 Overview of Treatment-Emergent Adverse Events (Japan SAF; $N=47$ )

\begin{tabular}{|c|c|c|c|c|}
\hline \multirow[t]{2}{*}{ TEAEs } & \multicolumn{2}{|l|}{$\begin{array}{l}\text { Gilteritinib } \\
(n=33)\end{array}$} & \multicolumn{2}{|c|}{ Salvage chemotherapy $(n=14)$} \\
\hline & All TEAEs, $n(\%)$ & TEAEs/PY & All TEAEs, $n(\%)$ & TEAEs/PY \\
\hline Any TEAE & $33(100)$ & 53.84 & $13(93)$ & 93.08 \\
\hline Drug-related TEAE & $29(88)$ & 19.73 & $10(71)$ & 51.54 \\
\hline Serious TEAE & $24(73)$ & 4.49 & $1(7)$ & 0.77 \\
\hline Drug-related serious TEAE & $13(39)$ & 2.05 & $1(7)$ & 0.77 \\
\hline TEAE leading to death & $5(15)$ & 0.38 & 0 & 0 \\
\hline Drug-related TEAE leading to death & $2(6)$ & 0.22 & 0 & 0 \\
\hline TEAE leading to discontinuation & $4(12)$ & 0.43 & $1(7)$ & 2.31 \\
\hline $\begin{array}{l}\text { Drug-related TEAE leading to } \\
\text { discontinuation }\end{array}$ & $3(9)$ & 0.38 & $1(7)$ & 2.31 \\
\hline Grade $\geq 3$ TEAE & $32(97)$ & 14.05 & $13(93)$ & 45.38 \\
\hline Drug-related grade $\geq 3$ TEAE & $25(76)$ & 6.27 & $9(64)$ & 26.92 \\
\hline
\end{tabular}

Percentages were rounded to the nearest whole number

$P Y$ patient-year, $S A F$ safety analysis set, TEAE treatment-emergent adverse event 
Table 5 Treatment-emergent adverse events occurring in $\geq 30 \%$ of patients in any treatment arm (Japan SAF; $N=47)$
Table 6 Grade $\geq 3$ drug-related treatment-emergent adverse events occurring in $>5 \%$ of patients in any treatment arm $(\mathrm{SAF} ; N=355)$

\begin{tabular}{llllll}
\hline \multirow{2}{*}{ TEAE } & \multicolumn{2}{l}{ Gilteritinib $(n=33)$} & & \multicolumn{2}{l}{ Salvage chemotherapy $(n=14)$} \\
\cline { 2 - 3 } \cline { 5 - 6 } & All TEAEs, $n(\%)$ & TEAEs/PY & & All TEAEs, $n(\%)$ & TEAEs/PY \\
\hline Febrile neutropenia & $18(55)$ & 1.51 & & $4(29)$ & 3.85 \\
Increased aspartate aminotransferase & $17(52)$ & 3.24 & & $2(14)$ & 1.54 \\
Increased alanine aminotransferase & $15(46)$ & 2.38 & & $1(7)$ & 0.77 \\
Anemia & $13(39)$ & 1.08 & & $8(57)$ & 6.15 \\
Constipation & $13(39)$ & 0.97 & & $3(21)$ & 2.31 \\
Increased blood creatine phosphokinase & $13(39)$ & 3.19 & & 0 & 0 \\
Nausea & $12(36)$ & 1.03 & & $1(7)$ & 0.77 \\
Decreased platelet count & $13(39)$ & 1.41 & & $7(50)$ & 16.15 \\
Pyrexia & $11(33)$ & 0.92 & & $1(7)$ & 0.77 \\
Increased blood lactate dehydrogenase & $10(30)$ & 0.54 & & $1(7)$ & 0.77 \\
Decreased white blood cell count & $7(21)$ & 1.35 & & $5(36)$ & 4.62 \\
\hline
\end{tabular}

Percentages were rounded to the nearest whole number

$P Y$ patient-year, $S A F$ safety analysis set, TEAE treatment-emergent adverse event

\begin{tabular}{llllll}
\hline Grade $\geq 3$ drug-related TEAE & $\begin{array}{l}\text { Japanese patients } \\
(n=47)\end{array}$ & & \multicolumn{2}{l}{$\begin{array}{l}\text { All other patients } \\
(n=308)\end{array}$} \\
\cline { 2 - 3 } \cline { 5 - 6 } & $\begin{array}{l}\text { Gilteritinib } \\
(n=33)\end{array}$ & $\begin{array}{l}\text { Salvage } \\
\text { chemotherapy } \\
(n=14)\end{array}$ & $\begin{array}{l}\text { Gilteritinib } \\
(n=213)\end{array}$ & $\begin{array}{l}\text { Salvage } \\
\text { chemotherapy } \\
(n=95)\end{array}$ \\
\hline Febrile neutropenia & $12(36)$ & $3(21)$ & & $26(12)$ & $17(18)$ \\
Decreased platelet count & $9(27)$ & $4(29)$ & & $21(10)$ & $10(11)$ \\
Anemia & $8(24)$ & $6(43)$ & & $40(19)$ & $15(16)$ \\
Decreased white blood cell count & $6(18)$ & $4(29)$ & & $20(9)$ & $10(11)$ \\
Decreased neutrophil count & $6(18)$ & $2(14)$ & & $18(9)$ & $7(7)$ \\
Increased alanine aminotransferase & $6(18)$ & 0 & & $13(6)$ & $2(2)$ \\
Increased aspartate aminotransferase & $6(18)$ & 0 & & $14(7)$ & $1(1)$ \\
Neutropenia & $4(12)$ & $1(7)$ & & $17(8)$ & $7(7)$ \\
Increased blood creatine phosphokinase & $2(6)$ & 0 & $3(1)$ & 0 \\
Drug eruption & $2(6)$ & $1(7)$ & & 0 & 0 \\
Pneumonia & $2(6)$ & $1(7)$ & & $5(2)$ & $3(3)$ \\
Thrombocytopenia & $2(6)$ & $2(14)$ & $28(13)$ & $9(10)$ \\
Decreased appetite & 0 & $2(14)$ & 0 & 0 \\
Tumor lysis syndrome & 0 & $2(14)$ & 0 & 0 \\
\hline
\end{tabular}

Percentages were rounded to the nearest whole number

$S A F$ safety analysis set, TEAE treatment-emergent adverse event
28 days; range 1-214 days), hospitalizations due to TEAEs were less frequent ( $55 \%$ vs $76 \%$, respectively).

\section{Discussion}

The utility of targeting FLT3 mutations in AML has gained momentum based on findings from clinical trials [22, 25, 26, 28, 34] and the approval of FLT3-targeted agents [27, 35]. Results from the QuANTUM-R and ADMIRAL studies provide compelling evidence that targeting FLT3 improves response rate compared with $\mathrm{SC}$ in patients with $\mathrm{R} / \mathrm{R} F L T$ $3^{\text {mut+ }}$ AML $[22,28]$. However, it is important to note that the ADMIRAL trial enrolled patients with either FLT3-ITD and/or FLT3-TKD mutations due to gilteritinib's activity against both mutation types, and included patients who had relapsed within or after 6 months following initial therapy [28]. The QuANTUM-R trial only enrolled patients with FLT3-ITD mutations who had relapsed within 6 months of initial therapy [22]. While findings from single-arm studies of gilteritinib and quizartinib in Japanese R/R AML patients have been reported [29, 36], this is the first comparative 
evaluation of gilteritinib versus conventional SC in a Japanese R/R AML cohort.

In the overall study population, patients randomized to gilteritinib had significantly longer OS and numerically higher rates of CR/CRh compared with patients randomized to SC [28]. The Japanese subgroup exhibited a similar trend, with numerically longer $\mathrm{OS}$ and improved $\mathrm{CR} / \mathrm{CRh}$ rates compared with SC (49\% vs $13 \%$, respectively). In contrast to the overall study population (40\%), most Japanese patients $(60 \%)$ had been preselected for low-intensity chemotherapy. Overall, $79 \%$ of Japanese patients in the SC arm received low-intensity chemotherapy and none of these patients achieved CR/CRh. Findings related to safety and tolerability in the Japanese subgroup were generally consistent with those of the overall study population [28].

Exposure to gilteritinib was longer in Japanese patients (median, 5.1 months) than in the overall study population (median, 4.1 months) and a greater proportion of Japanese patients required dose reductions (39\%) or interruptions (67\%) compared with the overall study population (31\% and $50 \%$, respectively). The median duration of hospitalization was also longer in the Japanese subgroup (133 days) compared with the overall study population (28 days), which may partially stem from the fact that extended hospitalization does not necessarily increase medical expenses for Japanese patients eligible for the high-cost medical expense benefit issued by the Japan MHLW. Notably, oral gilteritinib therapy allows for outpatient treatment, which could potentially lower treatment costs and improve quality of life.

A phase 2 study of quizartinib in Japanese patients with R/R FLT3-ITD + AML reported a similar rate of CRc (54\%) to that observed with gilteritinib (58\%) in the Japanese subgroup from the ADMIRAL trial [36]. However, CRi was the most frequent response (48\%) with quizartinib in the Japanese phase 2 study, with no patients achieving CR [36]. In contrast, $24 \%$ of Japanese patients achieved CR with gilteritinib in the ADMIRAL trial. A higher incidence of QT prolongation (35\%) was observed with quizartinib [36] compared with gilteritinib $(<10 \%)$, whereas liver enzyme (AST/ALT) elevations occurred more frequently with gilteritinib (46-52\%) than with quizartinib $(<10-11 \%)$. Although Japanese patients treated with gilteritinib in the ADMIRAL trial had a higher incidence of grade $\geq 3$ febrile neutropenia (55\%) than those who received quizartinib in the Japanese phase 2 study ( $43 \%$ ), rates of grade $\geq 3$ anemia ( $33 \%$ vs $27 \%$ ) and thrombocytopenia (9\% vs 11\%) were similar [36].

As is the nature of subgroup analyses, this analysis was restricted to a small, rather homogenous patient subset, without adjustment for multiple comparisons. Baseline characteristics were less balanced in the Japanese subgroup compared with the overall study population. As most Japanese patients treated with SC were aged $>65$ years $(87 \%)$ and received low-intensity chemotherapy (79\%), a potential bias toward poorer outcomes with SC treatment in this Japanese subgroup cannot be ruled out. Thus, results from this analysis should be interpreted with caution.

Single-agent gilteritinib therapy improved response rates in Japanese patients with $\mathrm{R} / \mathrm{R} F L T 3^{\mathrm{mut}+} \mathrm{AML}$ and resulted in numerically longer survival compared with SC. The observed trends in treatment efficacy and safety are consistent with the overall ADMIRAL study population and confirm that gilteritinib is an important treatment option for the Japanese R/R FLT3 ${ }^{\text {mut+ }}$ AML population. Investigations of gilteritinib as part of induction/consolidation (NCT02236013 and NCT04027309) and as post-consolidation/post-HSCT maintenance therapy (NCT02927262 and NCT02997202) are ongoing.

Acknowledgements We would like to acknowledge all patients and their families for their participation in this study. In addition, we also acknowledge the investigators, coordinators, and site personnel involved in this study. This study was funded by Astellas Pharma, Inc. Medical writing/editorial assistance was provided by Patrick Tucker, $\mathrm{PhD}$, Kalpana Vijayan, PhD, and Elizabeth Hermans, PhD, from Peloton Advantage, LLC, an OPEN Health company, Parsippany, NJ, and funded by the study sponsor.

Author contributions NH from AP US, Inc. and MK from AP, Inc. contributed to the study design along with SM and TN. Data analyses were performed by QL, MK, EB, and $\mathrm{NH}$. All authors were involved in data acquisition and interpretation, and in the development and critical review of the manuscript draft.

Data availability Researchers may request access to anonymized participant level data, trial level data and protocols from Astellas sponsored clinical trials at www.clinicalstudydatarequest.com.

For the Astellas criteria on data sharing see: https://clinicalstudyda tarequest.com/Study-Sponsors/Study-Sponsors-Astellas.aspx.

\section{Declarations}

Conflict of interest SF has received personal fees from Astellas Pharma, Celgene, Chugai Pharmaceutical, Eisai, Kyowa Hakko Kirin, Nippon Shinyaku, Novartis, Ortho Clinical Diagnostics, Otsuka, and Pfizer, all outside the submitted work. SC has received financial support from Astellas Pharma, Ono Pharmaceutical Co, Kyowa-Kirin, Takeda Pharmaceuticals, Sanofi, Bristol-Myers Squibb, and Chugai Pharmaceutical. M Kizaki has received personal fees from BristolMyers Squibb, Celgene, Novartis Pharma, Janssen Pharma, and Sumitomo Dainippon Pharma, all outside the submitted work. M Kizaki also received grants and personal fees from Takeda, Kyowa Kirin, and Ono Pharmaceutical, as well as grants from Chugai Pharmaceutical and Daiichi-Sankyo, all outside the submitted work. TN has received personal fees from Eisai, Astellas Pharma, Nippon Shinyaku, BristolMyers Squibb, and Sysmex, all outside the submitted work. Y Maeda has received honoraria from Mundipharma, Kyowa-Kirin, BristolMyers Squibb, Chugai Pharma, Pfizer, Celgene, Novartis, and Pfizer. $Y$ Maeda also received research funding from Astellas Pharma, Bristol-Myers Squibb, Takeda, Kyowa Kirin, and Chugai Pharmaceutical. TS has received grants from Astellas Pharma during the conduct of the study. TS also received personal fees from Eisai, Novartis, Kyowa Kirin, Takeda Pharmaceutical, Celgene, Bristol-Myers Squibb, Ono Pharmaceutical, Pfizer, Janssen Pharmaceutical, and Otsuka Pharmaceutical, all outside the submitted work. KU has received grants 
and personal fees from Astellas Pharma, Alexion Pharmaceuticals, AbbVie, Gilead, SymBio Pharmaceuticals, Daiichi Sankyo, Otsuka Pharmaceutical, Novartis, Bristol-Myers Squibb, Ono Pharmaceutical, Celgene, Takeda Pharmaceutical, Nippon, Boehringer Ingelheim, and Kyowa Kirin. KU also received grants from Sumitomo Dainippon Pharma, Chugai Pharmaceutical, Janssen Pharmaceutical, Mundipharma, Astellas-Amgen-Biopharma, Apellis Pharmaceuticals, Nippon Shinyaku, and Pfizer. KU also received personal fees from Eisai, MSD, SymBio Pharmaceuticals, PharmaEssentia Corp., and Yakult Honsha, all outside the submitted work. YK received grants, personal fees, and other support from Pfizer; YK also received personal fees from Astellas and Symbio, all outside the submitted work. JK has received other support from Astellas during the conduct of the study; JK has also received grants and personal fees from Astellas outside the submitted work. M Kusano, QL, EB, and N Hasabou are all employees of Astellas Pharma. N Hosono, HY, NA, KA, KK, TH, MH, MN, TI, MO, and $\mathrm{Y}$ Morita have no financial relationships to disclose.

Open Access This article is licensed under a Creative Commons Attribution 4.0 International License, which permits use, sharing, adaptation, distribution and reproduction in any medium or format, as long as you give appropriate credit to the original author(s) and the source, provide a link to the Creative Commons licence, and indicate if changes were made. The images or other third party material in this article are included in the article's Creative Commons licence, unless indicated otherwise in a credit line to the material. If material is not included in the article's Creative Commons licence and your intended use is not permitted by statutory regulation or exceeds the permitted use, you will need to obtain permission directly from the copyright holder. To view a copy of this licence, visit http://creativecommons.org/licenses/by/4.0/.

\section{References}

1. Niino M, Matsuda T (2016) Type distribution of myeloid leukemia from Cancer Incidence in Five Continents Vol. X. Jpn J Clin Oncol 46(4):394

2. Miyawaki S (2012) Clinical studies of acute myeloid leukemia in the Japan Adult Leukemia Study Group. Int J Hematol 96(2):171-177

3. Breems DA, Van Putten WL, Huijgens PC et al (2005) Prognostic index for adult patients with acute myeloid leukemia in first relapse. J Clin Oncol 23(9):1969-1978

4. Ganzel C, Sun Z, Cripe LD et al (2018) Very poor long-term survival in past and more recent studies for relapsed AML patients: The ECOG-ACRIN experience. Am J Hematol 93:1074-1081

5. Kihara R, Nagata Y, Kiyoi H et al (2014) Comprehensive analysis of genetic alterations and their prognostic impacts in adult acute myeloid leukemia patients. Leukemia 28(8):1586-1595

6. Levis M (2017) Midostaurin approved for FLT3-mutated AML. Blood 129(26):3403-3406

7. Kiyoi H, Towatari M, Yokota S et al (1998) Internal tandem duplication of the FLT3 gene is a novel modality of elongation mutation which causes constitutive activation of the product. Leukemia 12(9):1333-1337

8. Sakaguchi M, Yamaguchi H, Kuboyama M et al (2019) Significance of FLT3-tyrosine kinase domain mutation as a prognostic factor for acute myeloid leukemia. Int J Hematol 110(5):566-574

9. Yamamoto Y, Kiyoi H, Nakano Y et al (2001) Activating mutation of D835 within the activation loop of FLT3 in human hematologic malignancies. Blood 97(8):2434-2439
10. Bacher U, Haferlach C, Kern W et al (2008) Prognostic relevance of FLT3-TKD mutations in AML: the combination matters-an analysis of 3082 patients. Blood 111(5):2527

11. Yokota S, Kiyoi H, Nakao M et al (1997) Internal tandem duplication of the FLT3 gene is preferentially seen in acute myeloid leukemia and myelodysplastic syndrome among various hematological malignancies. A study on a large series of patients and cell lines. Leukemia 11(10):1605-1609

12. Chevallier P, Labopin M, Turlure P et al (2011) A new leukemia prognostic scoring system for refractory/relapsed adult acute myelogeneous leukaemia patients: a GOELAMS study. Leukemia 25(6):939-944

13. Papaemmanuil E, Gerstung M, Bullinger L et al (2016) Genomic classification and prognosis in acute myeloid leukemia. N Engl J Med 374(23):2209-2221

14. Wattad M, Weber D, Dohner K et al (2017) Impact of salvage regimens on response and overall survival in acute myeloid leukemia with induction failure. Leukemia 31(6):1306-1313

15. Smith CC, Lin K, Stecula A et al (2015) FLT3 D835 mutations confer differential resistance to type II FLT3 inhibitors. Leukemia 29(12):2390-2392

16. Smith CC, Wang Q, Chin CS et al (2012) Validation of ITD mutations in FLT3 as a therapeutic target in human acute myeloid leukaemia. Nature 485(7397):260-263

17. Daver N, Schlenk RF, Russell NH et al (2019) Targeting FLT3 mutations in AML: review of current knowledge and evidence. Leukemia 33(2):299-312

18. Smith BD, Levis M, Beran M et al (2004) Single-agent CEP701, a novel FLT3 inhibitor, shows biologic and clinical activity in patients with relapsed or refractory acute myeloid leukemia. Blood 103(10):3669-3676

19. Fiedler W, Serve H, Dohner H et al (2005) A phase 1 study of SU11248 in the treatment of patients with refractory or resistant acute myeloid leukemia (AML) or not amenable to conventional therapy for the disease. Blood 105(3):986-993

20. Fischer T, Stone RM, Deangelo DJ et al (2010) Phase IIB trial of oral Midostaurin (PKC412), the FMS-like tyrosine kinase 3 receptor (FLT3) and multi-targeted kinase inhibitor, in patients with acute myeloid leukemia and high-risk myelodysplastic syndrome with either wild-type or mutated FLT3. J Clin Oncol 28(28):4339-4345

21. O'Farrell AM, Yuen HA, Smolich B et al (2004) Effects of SU5416, a small molecule tyrosine kinase receptor inhibitor, on FLT3 expression and phosphorylation in patients with refractory acute myeloid leukemia. Leuk Res 28(7):679-689

22. Cortes JE, Khaled S, Martinelli G et al (2019) Quizartinib versus salvage chemotherapy in relapsed or refractory FLT3-ITD acute myeloid leukaemia (QuANTUM-R): a multicentre, randomised, controlled, open-label, phase 3 trial. Lancet Oncol 20:984

23. Galanis A, Levis M (2015) Inhibition of c-Kit by tyrosine kinase inhibitors. Haematologica 100(3):e77-79

24. Lee LY, Hernandez D, Rajkhowa T et al (2017) Preclinical studies of gilteritinib, a next-generation FLT3 inhibitor. Blood 129(2):257-260

25. Mori M, Kaneko N, Ueno Y et al (2017) Gilteritinib, a FLT3/ AXL inhibitor, shows antileukemic activity in mouse models of FLT3 mutated acute myeloid leukemia. Invest New Drugs 35(5):556-565

26. Perl AE, Altman JK, Cortes J et al (2017) Selective inhibition of FLT3 by gilteritinib in relapsed or refractory acute myeloid leukaemia: a multicentre, first-in-human, open-label, phase 1-2 study. Lancet Oncol 18(8):1061-1075

27. Pharmaceutical Evaluation Division, Pharmaceutical Safety and Environmental Health Bureau. Ministry of Health, Labour and Welfare. Report on the Deliberation Results. https://www.pmda. go.jp/files/000228502.pdf. Accessed July 20, 2020. (2018) 
28. Perl AE, Martinelli G, Cortes JE et al (2019) Gilteritinib or chemotherapy for relapsed or refractory FLT3-mutated AML. N Engl J Med 381(18):1728-1740

29. Usuki K, Sakura T, Kobayashi Y et al (2018) Clinical profile of gilteritinib in Japanese patients with relapsed/refractory acute myeloid leukemia: an open-label phase 1 study. Cancer Sci 109(10):3235-3244

30. Murphy KM, Levis M, Hafez MJ et al (2003) Detection of FLT3 internal tandem duplication and D835 mutations by a multiplex polymerase chain reaction and capillary electrophoresis assay. J Mol Diagn 5(2):96-102

31. Levis M, Ravandi F, Wang ES et al (2011) Results from a randomized trial of salvage chemotherapy followed by lestaurtinib for patients with FLT3 mutant AML in first relapse. Blood 117(12):3294-3301

32. Jackson G, Taylor P, Smith GM et al (2001) A multicentre, open, non-comparative phase II study of a combination of fludarabine phosphate, cytarabine and granulocyte colony-stimulating factor in relapsed and refractory acute myeloid leukaemia and de novo refractory anaemia with excess of blasts in transformation. Br J Haematol 112(1):127-137
33. Cheson BD, Bennett JM, Kopecky KJ et al (2003) Revised recommendations of the International working group for diagnosis, standardization of response criteria, treatment outcomes, and reporting standards for therapeutic trials in acute myeloid leukemia. J Clin Oncol 21(24):4642-4649

34. Stone RM, Mandrekar SJ, Sanford BL et al (2017) Midostaurin plus chemotherapy for acute myeloid leukemia with a FLT3 mutation. N Engl J Med 377(5):454-464

35. PMDA Quizartinib Approval. https://www.pmda.go.jp/files/00023 5289.pdf. Accessed March 3, 2020

36. Takahashi T, Usuki K, Matsue K et al (2019) Efficacy and safety of quizartinib in Japanese patients with FLT3-ITD positive relapsed or refractory acute myeloid leukemia in an open-label, phase 2 study. Int J Hematol 110(6):665-674

Publisher's Note Springer Nature remains neutral with regard to jurisdictional claims in published maps and institutional affiliations. 